\title{
Equipo de fusión al vacío para la determinación cuantitativa del gas atrapado en piezas fundidas ${ }^{(*)}$
}

\author{
J.J. Hernández-Ortega*, R. Zamora*, F. Faura* y J. López ${ }^{*}$
}

Resumen

Las piezas obtenidas mediante procesos de fundición pueden contener cantidades importantes de gas ocluido, lo que podría afectar negativamente a las propiedades mecánicas de las piezas fabricadas. En este artículo se describe un equipo de fusión al vacío fabricado para la determinación de la cantidad de gas ocluido en piezas obtenidas mediante procesos de fundición. Se han obtenido correlaciones de ajuste que permiten el cálculo, con un buen grado de precisión, de la cantidad de gas ocluido en piezas fundidas en función de los incrementos de presión detectados por el equipo de vacío. Finalmente, se presentan, como aplicación del equipo, los resultados obtenidos en piezas fabricadas en una máquina industrial de fundición por inyección a presión. Se espera que el equipo desarrollado sea de gran utilidad para optimizar las condiciones de operación que reduzcan el aire atrapado en procesos de fundición.

\section{Vacuum furnace equipment for quantitative determination of trapped gases in castings}

\begin{abstract}
Parts manufactured with casting processes may trap substantial quantities of gas, which could harm the mechanical properties of the manufactured parts. In this work, a vacuum melting device manufactured to measure trapped gas in castings is described. Adjustment correlations, which accurately calculate trapped gas quantity as a function of the pressure increments measured by the vacuum equipment, are obtained. Finally, as an application of the equipment, we present the results obtained for several parts manufactured using a pressure die casting machine. It is expected that the vacuum melting device will be useful to optimize the operating conditions which reduce the trapped gas in casting processes.
\end{abstract}

Keywords

Porosity; Trapped gas; Vacuum furnace; Die casting.

\section{INTRODUCCIÓN}

Uno de los defectos más frecuentes que presentan las piezas fabricadas con procesos de fundición y que limita sus posibilidades de aplicación es la porosidad. La porosidad puede ser debida, entre otras causas, al atrapamiento de gases durante el llenado del molde o a la contracción del metal durante su solidificación. En algunos procesos de fundición como, por ejemplo, la fundición por inyección a presión, la porosidad final de la pieza se puede reducir significativamente, aunque no la cantidad de aire atrapado, ejerciendo una alta presión sobre el metal al final del proceso de inyección mejorando, de esta forma, las propiedades mecánicas más significativas. Sin embargo, cuando las piezas necesitan ser sometidas a procesos posteriores tales como soldadura o tratamientos térmicos, el gas atrapado a presión puede llegar a provocar durante su expansión la deformación de la pieza o graves defectos en la misma.

Son muchos los estudios realizados y los métodos utilizados para la evaluación de la porosidad en piezas fabricadas por fundición. Entre los que utilizan ensayos no destructivos podemos destacar los métodos basados en la inspección visual ${ }^{[1]}$, el radiografiado ${ }^{[2]}$, la tomografía asistida por ordenador ${ }^{[3]}$, el uso de ultrasonidos $^{[4]}$, el estudio de frecuencias de resonancia ${ }^{[5]}$ o el método gravimétrico ${ }^{[6-11]}$. Entre los que utilizan métodos destructivos destacan el análisis de imagen de distintas secciones de la pieza ${ }^{[12}$ y 13$]$ y el calentamiento

(•) Trabajo recibido el día 1 de febrero de 2008 y aceptado en su forma final el día 25 de septiembre de 2008.

* Dept. de Ingeniería de Materiales y Fabricación, ETSII, Universidad Politécnica de Cartagena, C/ Doctor Fleming, s/n. E-30202 Cartagena,

España. e-mail: rosendo.zamora@upct.es. 
de las muestras para provocar la expansión del gas atrapado y el posterior empleo del método gravimétrico ${ }^{[14]}$. Sin embargo, ninguno de estos métodos permite determinar la cantidad de gas atrapado en la pieza ya que, generalmente, la presión del gas ocluido en la pieza es desconocida. Para resolver este inconveniente, se puede emplear la técnica de fusión al vacío $^{[8 \text { y } 15-17]}$, en la que la pieza, cuya cantidad de gas atrapado se quiere medir, se funde en el interior de una cámara de vacío estanca bajo condiciones de presión, volumen y temperatura controladas. Durante la fusión de la pieza el gas atrapado se libera provocando incrementos de presión en la cámara. El principio básico para la medición de la cantidad de gas atrapado consiste en relacionar las variaciones de presión en la cámara con la cantidad de gas liberado tras la fusión de la pieza. Aunque existen trabajos experimentales en los que se describe el diseño de un sistema de fusión al vacío ${ }^{[15]}$ y otros en los que se presentan resultados para piezas fabricadas con zamak$3^{[16]}$ o aluminio ${ }^{[8 \text { y } 16]}$, la información sobre el diseño, fabricación y funcionamiento de estos equipos, así como la verificación y obtención de las curvas o correlaciones de ajuste es escasa.

En este trabajo se realiza una descripción detallada de un equipo de fusión al vacío, exponiendo la metodología utilizada y los resultados obtenidos en la verificación de su funcionamiento. También se obtienen curvas y correlaciones de ajuste para calcular con precisión la cantidad de gas atrapado en piezas fundidas. Las correlaciones se obtienen a partir de los incrementos de presión detectados por el equipo tras la liberación de cantidades conocidas de gas en el interior de la cámara de vacío. La aplicación del equipo a varias piezas fabricadas en una máquina industrial de fundición por inyección a presión muestra las posibilidades que ofrece su uso para la optimización del proceso de fundición.

\section{DESCRIPCIÓN DEL EQUIPO DE FUSIÓN AL VACÍO}

Existen dos configuraciones básicas de equipos de fusión al vacío: (1) la cámara de vacío se encuentra situada en el interior del sistema de fusión ${ }^{[8,15 \text { y } 17]}$ y (2) el sistema de fusión forma parte de la cámara de

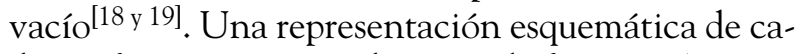
da configuración se puede ver en la figura 1 . Aunque ambas configuraciones presentan sus propias ventajas e inconvenientes, la diversidad de problemas que pueden surgir con la configuración (2) y su relevancia (deposición en las partes eléctricas del horno de metales con baja presión de vapor presentes en la pieza o la presencia de un mayor número de elementos en la cámara de vacío, tales como aislantes térmicos o componentes eléctricos y mecánicos que aumentan la variabilidad de las medidas de presión,

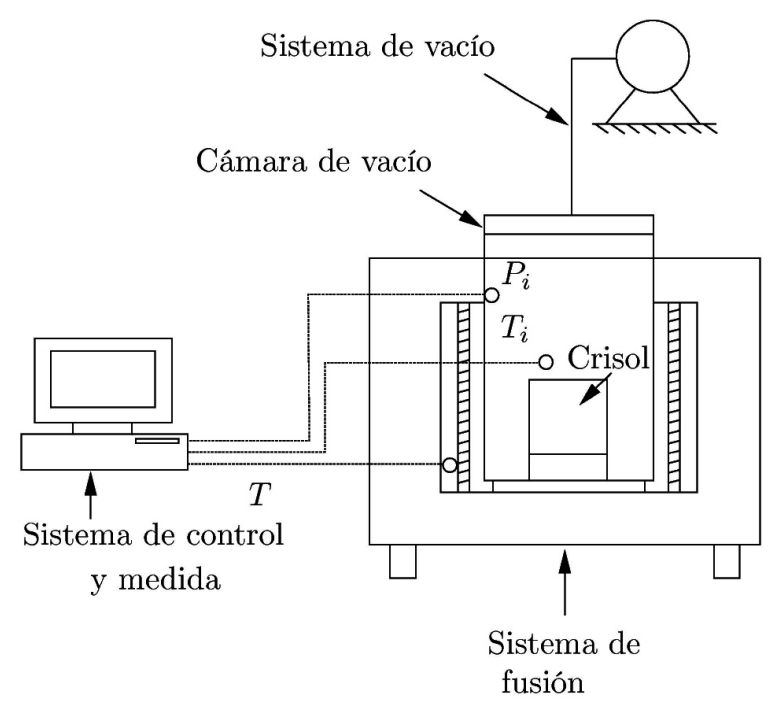

(a)

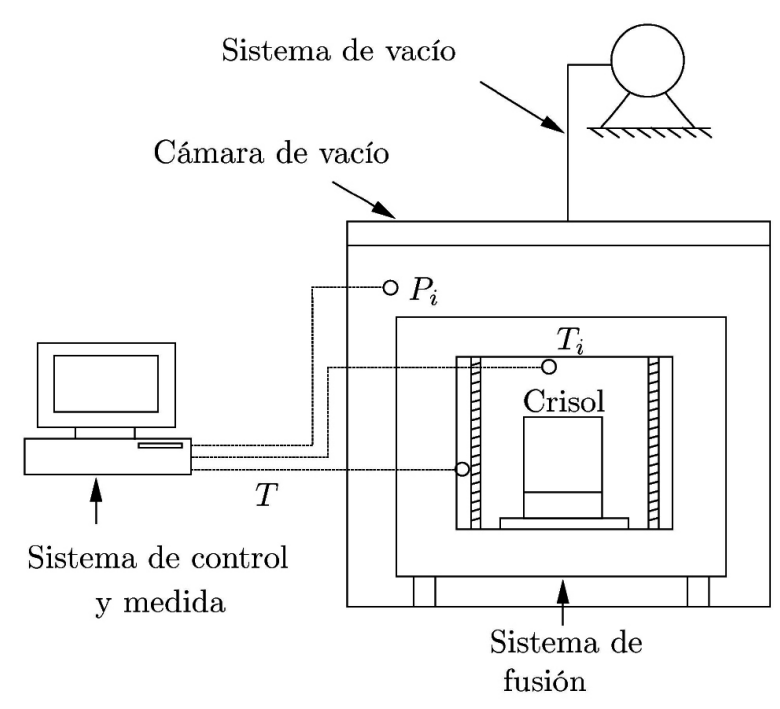

(b)

Figura 1. Configuraciones básicas de los sistemas de fusión al vacío. a) Cámara de vacío dentro del horno de fusión. b) Horno de fusión dentro de la cámara de vacío.

Figure 1. Basic layouts of the vacuum melting equipment. a) Vacuum chamber inside the furnace. b) Furnace inside the vacuum chamber. 
temperatura y volumen en el interior de la cámara de vacío) hacen que la configuración (1) sea la mejor opción para este tipo de equipos.

En la figura 2 se muestra un esquema del equipo fabricado que consta de tres sistemas principales: sistema de vacío, sistema de fusión y sistema de control, medida y registro. Para mejorar la estanquidad del sistema de vacío, principal problema cuando la configuración es de tipo (1) y cuando se pretenden alcanzar elevadas temperaturas, se ha optado por emplear una junta de material elastómero similar a la utilizada por Robla et al. ${ }^{[15]}$, pero incorporando un sistema de refrigeración por agua en circuito cerrado de las zonas de cierre (en la tapa de la cámara de vacío) así como pantallas de protección que evitan el calentamiento excesivo por radiación en la zona de la junta (Fig. 2 b)). La junta de material elastómero mejora la operatividad del equipo diseñado por Wang et al..$^{[17]}$, mientras que la refrigeración de la misma y su protección frente a la radiación permite el aumento de la temperatura de trabajo máxima alcanzada por Robla et al. ${ }^{[15]}$, pudiendo ser empleado de esta forma para la fusión de aleaciones de aluminio.

A continuación se describen con detalle los distintos sistemas enumerados anteriormente.

\subsection{Sistema de vacío}

El sistema de vacío (Fig. 2 a)) está formado por la cámara de vacío, como elemento principal, junto con los sistemas de cierre, válvulas, bombas de vacío y sistemas de fijación necesarios. La cámara fabricada (Fig. 2 b)) tiene un cuerpo cilíndrico fabricado en acero inoxidable AISI $316 \mathrm{~L}$ de $3 \times 10^{-3} \mathrm{~m}$ de espesor, con fondo ciego y tapa superior extraíble para permitir el acceso a la cámara. El volumen total de la cámara de vacío es de $11,248 \times 10^{-3} \mathrm{~m}^{3}$. Para evitar deformaciones del cuerpo cilíndrico de la cámara como consecuencia del calentamiento, ésta se ha reforzado con anillos rigidizadores en su interior. La hermeticidad del cierre del cuerpo con la tapa se consigue mediante el uso de dos juntas de Vitón ${ }^{\mathrm{TM}}$ (elastómero con alta resistencia al calor $(473 \mathrm{~K})$ fabricado por la empresa Dupont Dow) comentadas anteriormente (véase el detalle de la Fig. 2 b)). Entre la tapa superior extraíble y la parte superior del crisol se encuentran dispuestas varias pantallas metálicas de $3 \times 10^{-3} \mathrm{~m}$ de espesor, cuya finalidad es aislar térmicamente la zona de cierre del calor emitido por radiación. Además, la tapa de la cámara de vacío está dotada de un sistema de refrigeración por agua en circuito cerrado para evitar el calentamiento excesivo de las juntas y del transductor de presión. En la tapa, se encuentran disponibles tres puertos de acceso al interior de la cámara, dos para la conexión de termopares y el tercero para la conexión con las bombas de vacío y el transductor de presión de la cámara.

El sistema de vacío cuenta con una bomba mecánica rotatoria y una bomba de difusión de aceite conectadas en serie que permiten alcanzar presiones de vacío en la cámara de hasta $5 \times 10^{-6}$ torr. La conexión de las bombas con la cámara de vacío está regulada mediante electroválvulas gobernadas por el sistema de control. El sistema se ha diseñado para que permita detectar liberaciones de volumen de gas, referido a temperatura ambiente y presión atmosférica, igual o superiores a $0,1 \times 10^{-6} \mathrm{~m}^{3} \cdot \mathrm{s}^{-1}$.

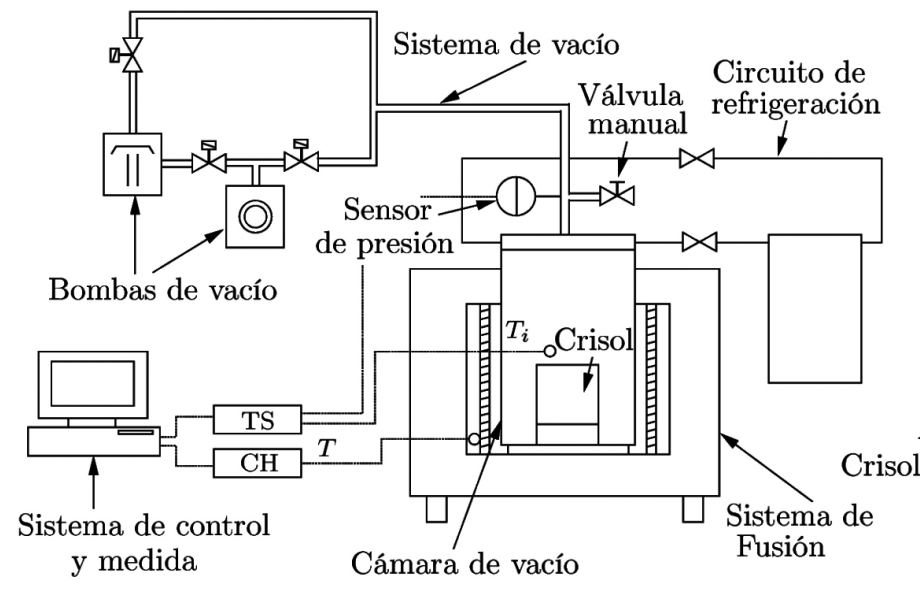

(a)

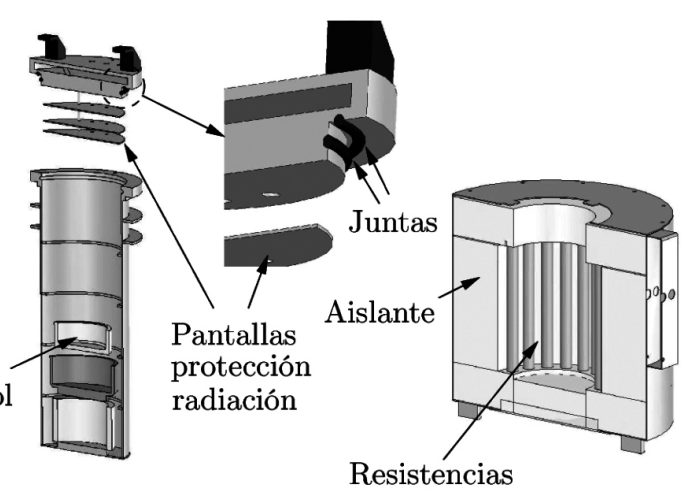

(b) (c)

Figura 2. a) Esquema del equipo de fusión al vacío. b) Cámara de vacío. c) Horno de fusión.

Figure 2. a) Schematic representation of the vacuum melting equipment. b) Vacuum chamber. c) Furnace. 
En el fondo del cilindro de la cámara va alojado un envase metálico, soportado por un anillo cerámico, donde se sitúa el crisol que contendrá la pieza a fundir. En los ensayos se utiliza un crisol con medidas de $0,135 \mathrm{~m}$ de diámetro y $0,205 \mathrm{~m}$ de altura, fabricado en titanato de aluminio, debido a su buen comportamiento térmico y a la escasa adherencia con las aleaciones de aluminio.

\subsection{Sistema de fusión}

El sistema de fusión está formado básicamente por el horno eléctrico de fusión (Fig. 2 a) y c)). El cuerpo del horno es de forma cilíndrica con una cavidad interior en la que va alojada la cámara de vacío y alrededor de la cual se disponen de forma radial 18 resistencias eléctricas de hilo distribuidas en tres grupos. Las resistencias están fabricadas en hilo de aleación Kanthal ${ }^{\mathrm{TM}}$ A1 de $1,8 \times 10^{-3} \mathrm{~m}$ arrollado en espiral sobre tubos cerámicos. Dispone de cuatro puertos laterales, utilizando los tres primeros para alojar las conexiones eléctricas de alimentación del horno y el cuarto para la ubicación del termopar de control del horno. Utiliza alimentación trifásica de $220 \mathrm{~V}$ y posee una potencia por fase de $3399 \mathrm{~W}$ siendo capaz de suministrar potencia suficiente para calentar la cámara de vacío y permitir la fusión de piezas de aleaciones de aluminio, alcanzándose en la cámara de vacío temperaturas de hasta $1.150 \mathrm{~K}$ con relativa facilidad.

\subsection{Sistema de control, medida y gestión}

El sistema de control, medida y gestión del equipo está formado, principalmente, por transductores de presión y de temperatura, el controlador del horno, una tarjeta de adquisición de datos y una aplicación informática de control y gestión integrada. Este sistema está centralizado en un ordenador PC y permite el control del proceso, tanto de forma manual como automática actuando sobre las bombas y electroválvulas, así como medir, visualizar y registrar las magnitudes de interés del proceso (temperatura y presión de la cámara de vacío, fundamentalmente).

\section{VERIFICACIÓN Y AJUSTE DEL EQUIPO}

La utilización del equipo de fusión al vacío requiere la realización previa de una serie de ensayos que permitan verificar el correcto funcionamiento del siste- ma y establecer curvas de ajuste para determinar con precisión la cantidad de gas liberado durante la fusión de una pieza en función del incremento de presión registrado por el equipo.

\subsection{Verificación del funcionamiento del equipo y obtención de curvas de ajuste}

La verificación del funcionamiento del equipo de fusión al vacío se efectúa comprobando, en primer lugar, la estanquidad del sistema a diferentes temperaturas y realizando, en segundo lugar, liberaciones de cantidades conocidas de aire a temperaturas y presiones fijadas en el interior de la cámara de vacío. Dichas liberaciones se realizan desde el exterior mediante el uso de diez probetas de diferentes volúmenes, fabricadas en acero inoxidable y conectadas al sistema de vacío a través de la válvula de mando manual (Figs. 2 a) y 3). Cada probeta está formada por un tubo de acero inoxidable, cerrado por un extremo, que se acopla mediante una brida y su correspondiente junta al sistema de fusión al vacío a través de una válvula. De este modo, mediante la apertura manual de la válvula se libera en la cámara una cantidad de aire conocida. En la figura 3 se pueden ver dichas probetas y su conexión a la cámara de vacío. En la tabla I se presentan los volúmenes correspondientes a cada probeta.

Considerando que, tras la liberación del gas de la probeta, éste formará una mezcla homogénea en la cámara a la temperatura media, $T_{c}$, y que el comportamiento del gas es ideal, se puede obtener la siguiente

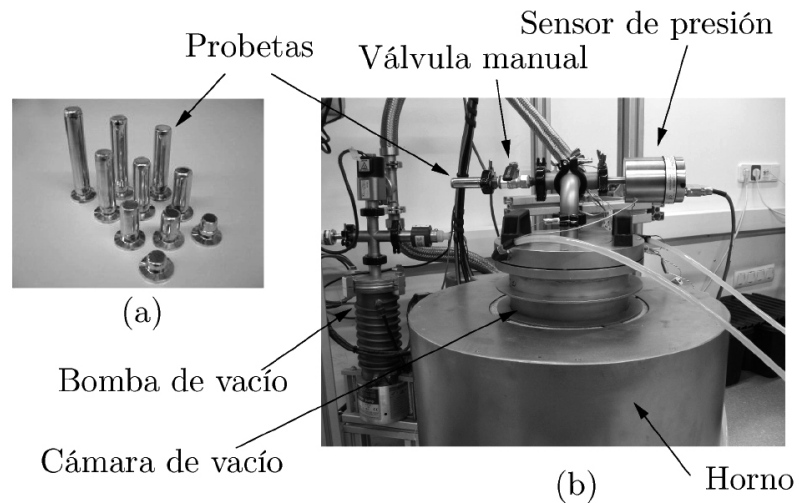

Figura 3. a) Probetas de acero inoxidable fabricadas para el ajuste del equipo y b) disposición de las mismas durante la liberación del aire.

Figure 3. a) Stainless steel test tubes constructed for adjusting the equipment and $b$ ) their localisation during air release. 
Tabla I. Volumen de las diferentes probetas de acero inoxidable

Table I. Volume of the different stainless steel test tubes

\begin{tabular}{cc}
\hline & $\begin{array}{c}\text { Volumen de la } \\
\text { probeta }\left(\mathbf{x ~ 1 0}^{-6} \mathrm{~m}^{3}\right)\end{array}$ \\
\hline 01 & 5,93 \\
02 & 6,38 \\
03 & 8,36 \\
04 & 10,42 \\
05 & 12,54 \\
06 & 14,26 \\
07 & 16,36 \\
08 & 18,49 \\
09 & 20,43 \\
10 & 22,86 \\
\hline
\end{tabular}

expresión para el incremento de presión $\Delta p$ experimentado en la cámara de vacío:

$$
\Delta p=V_{p} T_{c} \frac{\rho_{\mathrm{a}} R}{M V_{c}}(1-\xi)
$$

donde, $\xi=\frac{M V_{c} P_{c}+\rho_{a} R T T_{c} V_{p}}{\rho_{a} R T_{c}\left(V_{c}+V_{p}\right)}, V_{p}$ es el volumen de gas liberado por la probeta, $\rho_{\mathrm{a}}$ es la densidad del aire en la probeta (la temperatura y presión inicial del gas en la probeta es $298 \mathrm{~K}$ y 1 atmósfera, respectivamente), $\mathrm{M}$ el peso molecular del aire, $V_{c}$ el volumen de la cámara y $R$ la constante de los gases perfectos. Puede comprobarse que el parámetro $\xi$ es prácticamente nulo (el valor máximo que puede alcanzar para el rango de condiciones de operación consideradas en este trabajo es del orden de 0,009), por lo que la ecuación (1) quedará reducida, con un buen grado de aproximación, a la siguiente expresión:

$$
\Delta p \approx V_{p} T_{c} \frac{\rho_{\mathrm{a}} R}{M V_{c}}
$$

Las diferentes temperaturas de los ensayos se han establecido fijando la temperatura en la zona del crisol, $T_{i}$ (en la figura 2 puede verse la localización del sensor de temperatura en la zona del crisol). Los valores de $T_{i}$ elegidos han sido 298, 633, 933 y $1.050 \mathrm{~K}$. Debe tenerse en cuenta que la liberación del gas se realiza con las bombas de vacío desconectadas, tras haber efectuado la desgasificación de la cámara y con el horno encendido para mantener constante la temperatura,
$T_{i}$. También, debe tenerse en cuenta que, aunque las liberaciones de gas se realicen cuando la temperatura media de la cámara, $T_{c}$, se haya estabilizado debido a los altos gradientes térmicos existentes en la cáma$\mathrm{ra}^{[20]}$, dicha temperatura no coincidirá (excepto en el caso en el que el ensayo se realice a temperatura ambiente) con la temperatura, $T_{i}$, en la zona del crisol (la parte superior de la cámara está refrigerada y alejada de las resistencias del horno). Las liberaciones se han realizado para las temperaturas mencionadas con el objetivo de cubrir un rango de trabajo lo más amplio posible. El rango de presiones de trabajo (nivel de vacío) se ha elegido entre 0,5 y 5 torr.

En la figura 4 se representa, para las diferentes probetas, el incremento de presión, $\Delta p$, que se produce en la cámara de vacío tras la liberación del gas cuando el ensayo se realiza a temperatura ambiente, es decir con el horno apagado, y con la presión inicial de la cámara igual a 0,5 torr. Cada punto representado corresponde al valor medio de los incrementos de presión registrados tras repetir varias veces la liberación del gas contenido con la misma probeta (la diferencia máxima registrada para el incremento de presión medido para una misma probeta es de 0,010

$\Delta p($ torr $)$

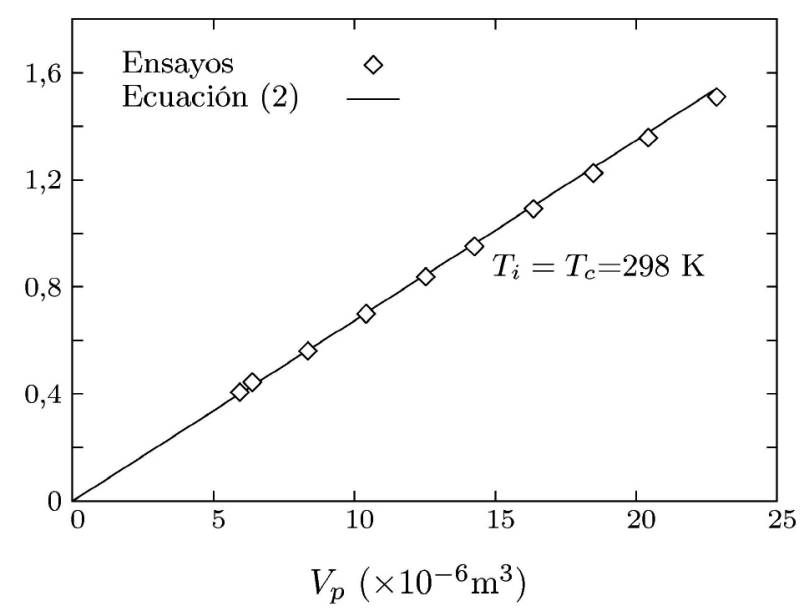

Figura 4. Comparación entre los resultados experimentales y teóricos del incremento de presión correspondiente a la liberación del aire contenido, a temperatura ambiente y presión atmosférica, en las diferentes probetas de acero inoxidable.

Figure 4. Comparison between experimental and theoretical results of pressure increase corresponding to the release of the air contained, at room temperature and atmospheric pressure, in the different stainless steel test tubes. 
torr). Los resultados correspondientes a la ecuación (2) también se representan en la figura, con línea de trazo continuo. Puede observarse que existe un excelente grado de concordancia entre los valores obtenidos experimentalmente y los correspondientes a la ecuación (2).

En la figura 5 se representan los valores del incremento de presión, $\Delta p$, obtenidos para diferentes condiciones iniciales de presión y temperatura en la cámara. Sigue observándose, para el rango de presiones elegido ( 0,5 y 5 torr $)$, un excelente grado de linealidad entre el incremento de presión registrado y el volumen de gas liberado. Conviene destacar que, a diferencia del caso de la figura 4, en el que el ensayo se realiza a temperatura ambiente, la temperatura media de la mezcla, $T$, no coincidirá con la temperatura $T_{i}$ en la zona del crisol. En estos casos, la temperatura media, $T_{c}$, se ha calculado a partir de la pendiente de las líneas de regresión obtenidas de los datos experimentales. En la figura 5 se muestran dichas líneas de regresión para la presión inicial de 0,5 torr, junto con la temperatura media, $T_{c}$, correspondiente. Obsérvese que el incremento de presión, $\Delta p$, experimentado para una misma probeta aumenta con la

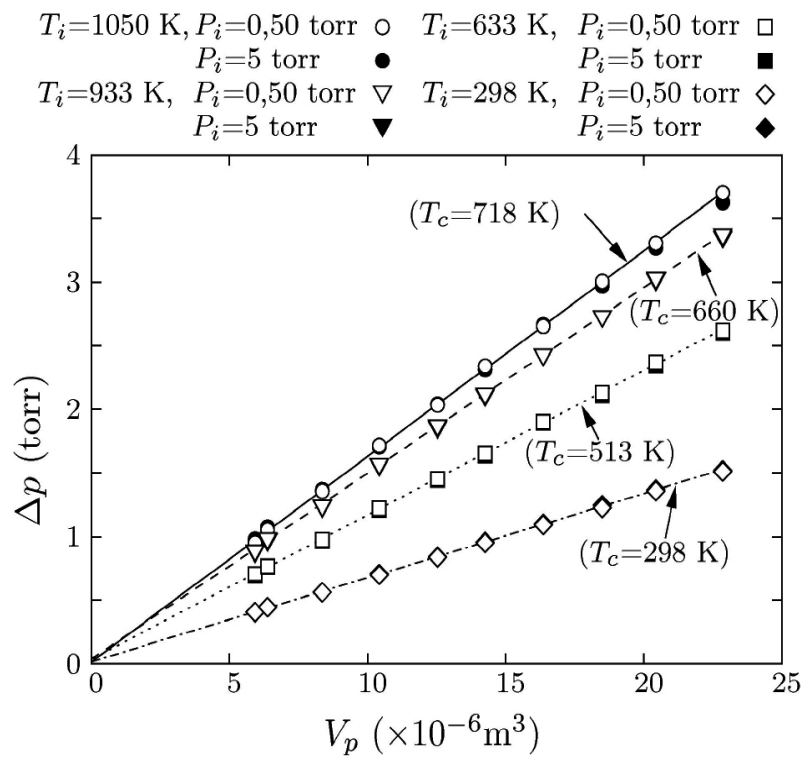

Figura 5. Incremento de presión en la cámara de vacío en función del volumen de aire, a temperatura ambiente y presión atmosférica, liberado para distintas temperaturas y presiones de la cámara de vacío, y curvas de ajuste obtenidas.

Figure 5. Pressure increase in the vacuum chamber as a function of the gas volume, at room temperature and atmospheric pressure, released for different temperatures and pressures in the vacuum chamber, and fitting curves obtained. temperatura media, $T_{\mathfrak{c}}$, del gas en la cámara de vacío, lo que concuerda con la expresión analítica de la ecuación (2).

Debe mencionarse que las líneas de regresión mostradas en la figura 5, además de servir para verificar el funcionamiento del equipo, también permitirán determinar, siguiendo el procedimiento descrito en la siguiente sección, la cantidad de gas ocluido en una pieza. La validez y precisión de dichas líneas de regresión será evaluada en la Sección 4.1 para la medida de gas ocluido en piezas de una aleación de aluminio.

\subsection{Procedimiento de ensayo para piezas de aluminio}

En condiciones reales de operación (es decir, cuando se pretende determinar la cantidad de gas atrapado en una pieza fabricada utilizando técnicas de fundición) es muy difícil conocer con precisión la temperatura exacta y el instante justo en el que la pieza se fundirá y se liberará el gas atrapado en ella. Téngase en cuenta que si la liberación del gas se produce en un instante en el que la temperatura en el interior de la cámara aún no está estabilizada, será muy difícil relacionar con precisión el incremento de presión detectado con la cantidad de gas ocluido en la pieza a través de las curvas de ajuste obtenidas en la sección anterior. En lo que sigue se describirá brevemente el procedimiento de ensayo utilizado para conseguir que la temperatura media de la cámara sea lo más estable posible, justo en el instante en el que se inicie la liberación del gas atrapado y la fusión de la pieza.

En primer lugar, se incrementa lentamente la temperatura, $T_{i}$, hasta alcanzar un valor de $633 \mathrm{~K}$, suficientemente alejado de la temperatura de fusión de la aleación de aluminio empleada para fabricar las piezas ensayadas en este trabajo. Durante todo este tiempo, la bomba de vacío se mantiene conectada para la desgasificación de la cámara. A continuación, se mantiene la temperatura de consigna del horno constante con la bomba de vacío conectada durante tres horas, aproximadamente, para estabilizar la temperatura en el interior de la cámara y conseguir una mejor desgasificación de todo el sistema. Transcurrido este tiempo, se desconecta la bomba de vacío para mantener completamente estanca la cámara y se incrementa, rápidamente y de forma controlada, la temperatura de consigna del horno hasta $1.123 \mathrm{~K}$ a una velocidad de $50 \mathrm{~K} \mathrm{~min}^{-1}$. A continuación, se mantiene dicha temperatura hasta el final del ensayo para conseguir, como se indicó anteriormente, que la temperatura en la cámara sea lo más estable posible en el momento de la liberación del gas ocluido. 


\section{APLICACIÓN DEL EQUIPO PIEZAS DE ALUMINIO}

A continuación se aplica el equipo, siguiendo el procedimiento descrito en la sección anterior, a dos tipos de piezas: piezas construidas con un volumen de gas ocluido conocido (Sección 4.1) y piezas obtenidas con un proceso de fundición por inyección a presión (Sección 4.2). En ambos casos, el material de las piezas es una aleación de aluminio EN AC-44100 (L2520), cuyo análisis de colada y temperatura de fusión se muestran en la tabla II.

\subsection{Piezas de aluminio con volumen conocido de gas ocluido}

El procedimiento de ensayo de la sección anterior se aplica a 10 piezas de aluminio con una cavidad interna de volumen similar al de las probetas de acero inoxidable (véase el esquema de la figura 6), lo que permitirá evaluar la precisión del equipo de fusión construido para medir el gas ocluido en piezas de aluminio. En cada pieza se mecaniza una cavidad de volumen conocido que posteriormente es sellada mediante una tapa soldada del mismo material. Con la finalidad de que el aire en el interior de la pieza se encuentre en condiciones de temperatura y presión conocidas, las piezas se taladran por su parte inferior comunicando la cavidad con el exterior. Posteriormente, cuando la temperatura de la pieza alcanza la temperatura ambiente, el orificio taladrado se sella con un tornillo. En la figura 6 b) se muestra la radiografía de una de las piezas de aluminio construidas.

En la figura 7 se representa la temperatura, $T_{i}$, que hay en la zona del crisol cuando se produce la liberación del gas ocluido en cada una de las piezas ensayadas siguiendo el procedimiento de la sección anterior. Se observa que dicha temperatura tiene un valor más o menos constante, en torno a $1.050 \mathrm{~K}$. Obsérvese que esta temperatura coincide con la

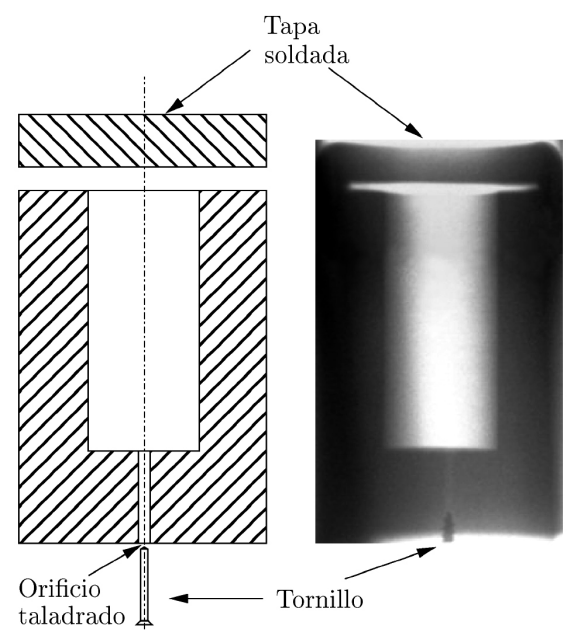

(a)

(b)

Figura 6. a) Esquema y b) radiografía de una de las piezas de aluminio utilizadas en el ajuste del equipo.

Figure 6. a) Schematic representation and b) $X$-ray of one of the aluminium parts used in equipment adjustment.

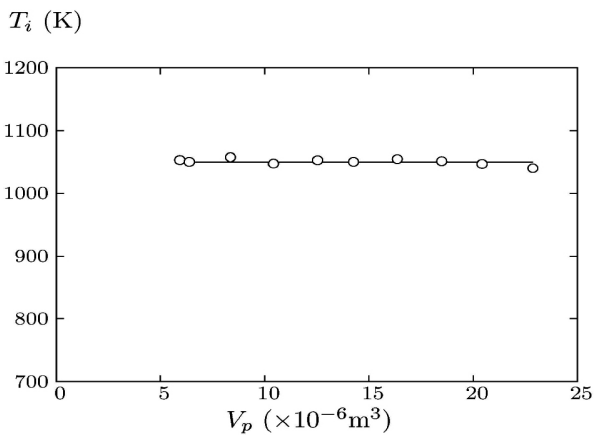

Figura 7. Temperatura en el crisol, $T_{i}$, en el momento de la liberación del gas ocluido en las piezas de aluminio.

Figure 7. Crucible temperature $T_{j}$ at the instant at which the occluded gas in the aluminium parts is released.

Tabla II. Análisis de colada e intervalo de temperaturas de solidificación de la aleación de aluminio EN AC-44100

Table II. Casting analysis and freezing temperature range of the EN AC-44100 aluminium alloy

\section{Composición química}

\begin{tabular}{cccccccccccc}
\hline Al & $\mathrm{Si}$ & $\mathrm{Fe}$ & $\mathrm{Cu}$ & $\mathrm{Mn}$ & $\mathrm{Mg}$ & $\mathrm{Ni}$ & $\mathrm{Zn}$ & $\mathrm{Ca}$ & $\mathrm{Pb}$ & $\mathrm{Sn}$ & $\mathrm{Ti}$ \\
\hline 88,06 & 11,72 & 0,14 & $<0,01$ & 0,01 & 0,01 & $<0,01$ & $<0,01$ & 0,001 & $<0,01$ & $<0,01$ & 0,01 \\
\hline
\end{tabular}

Intervalo de solidificación: 843 - 857 K 
temperatura (la de mayor valor) de una de las curvas de ajuste mostradas en la figura 5. Debe mencionarse que, aunque la temperatura de consigna del horno se mantenga constante después de la fase de calentamiento rápido, la temperatura en la zona del crisol seguirá aumentando ligeramente durante un periodo relativamente largo, hasta que se alcance completamente la estabilidad térmica de toda la cámara de vacío. En cualquier caso, se ha observado que en los 10 casos ensayados, las liberaciones se producen después de la fase de calentamiento rápido y durante el periodo en el que el incremento de la temperatura en la zona del crisol es relativamente pequeño, como puede observarse en la figura $8 \mathrm{a}$ ), donde, a modo de ejemplo, se representa la temperatura, $T_{i}$, y la presión en la cámara de vacío para tres de las piezas ensayadas, que corresponden a las piezas con una cavidad interna de volumen similar al de las probetas de acero inoxidable 01, 03 y 08 (Tabla I). Pueden observarse, marcados con flechas, los instantes en los que se inicia, en cada una de las piezas, la liberación del gas ocluido. Nótese que las pendientes del último tramo de las curvas de temperatura de los casos representados en dicha figura son relativamente pequeñas. Como ejemplo, en la Fig. 8 b) se representa, ampliado, el registro de presión correspondiente al caso 01 de la Fig. 8 a), indicando sobre la misma el procedimiento de cálculo del incremento de presión correspondiente (obsérvese que para este cálculo se ha tenido en cuenta la pendiente de la curva de presión existente en la cámara, justo, antes de que se produzca la liberación del gas).

Los resultados del incremento de presión correspondiente a las piezas de aluminio, calculado como se acaba de mencionar, se representan en la figura 9, con símbolo “ $\square$ ”, y se comparan con los representados en la figura 5, obtenidos en la verificación del equipo, para una temperatura. $T_{i}$, completamente estabilizada e igual a $1.050 \mathrm{~K}$ (símbolo 'o' en la figura 9). Para evaluar la influencia de la inestabilidad térmica en la cámara en el instante en el que se produce la liberación del gas ocluido en las piezas de aluminio, en la figura 9 se incluyen nuevos resultados realizados con las 10 probetas de acero inoxidable y siguiendo el mismo procedimiento de ensayo utilizado para las piezas de aluminio. Para ello, el aire de las probetas de acero inoxidable se libera, justo, en el momento en el que la temperatura del crisol alcanza el valor $T_{i}$ $=1.050 \mathrm{~K}$ tal y como ocurrió con las piezas de aluminio (es decir, justo, en el instante en el que se esperaría que se liberase el gas ocluido en una pieza de aluminio). Puede observarse en la figura que las diferencias entre los resultados así obtenidos (símbolo '+') y los correspondientes a los de la figura 5 (símbolo

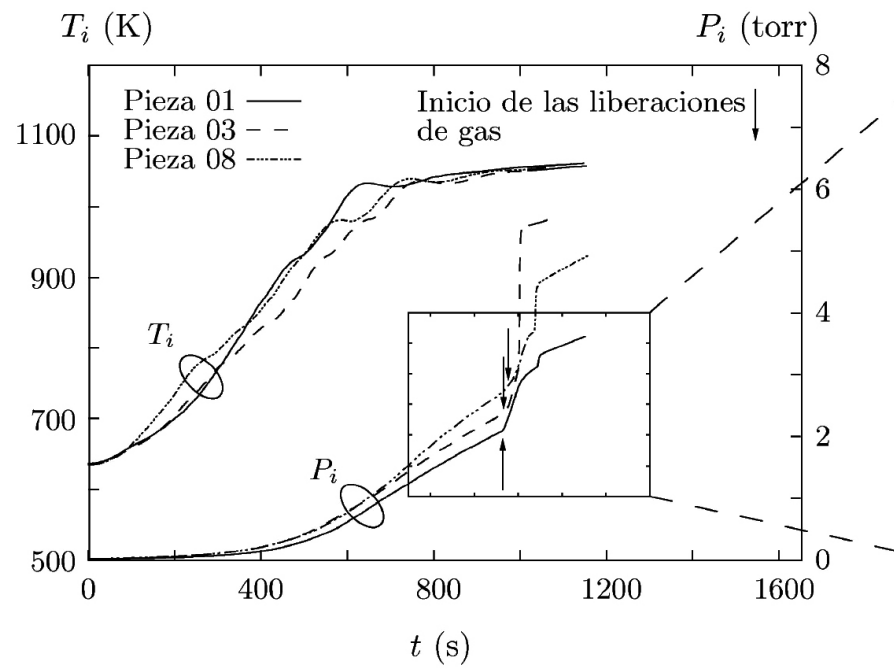

(a)

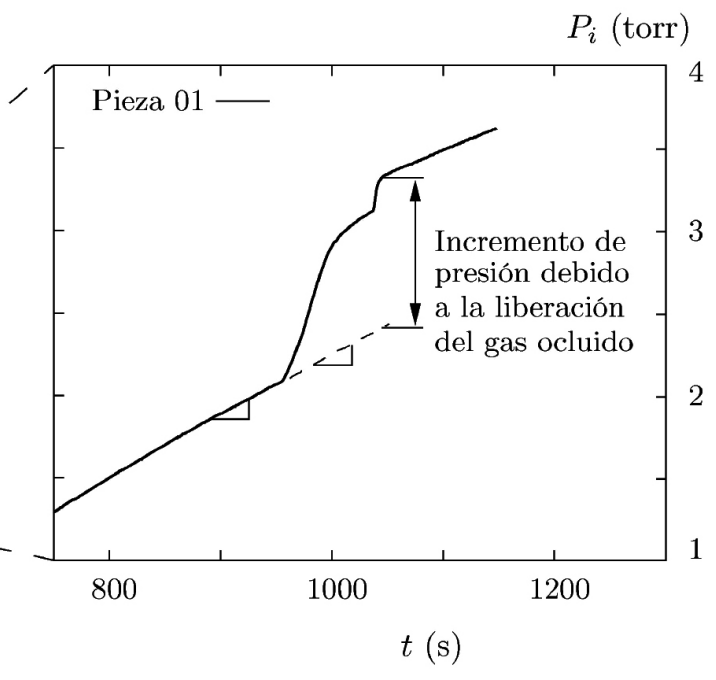

(b)

Figura 8. a) Registro de presión en la cámara y temperatura en el crisol, $T_{i}$, durante la etapa final del ensayo de tres de las piezas de aluminio construidas. b) Cálculo del salto de presión correspondiente a la liberación del aire en la pieza de aluminio con volumen equivalente al de la probeta de acero inoxidable 01.

Figure 8. a) Chamber pressure and crucible temperature record during the last step of testing three of the aluminium parts manufactured. b) Calculation of the pressure increase corresponding to the air released from the aluminium part with a volume equivalent to that of the stainless steel test tube 01. 


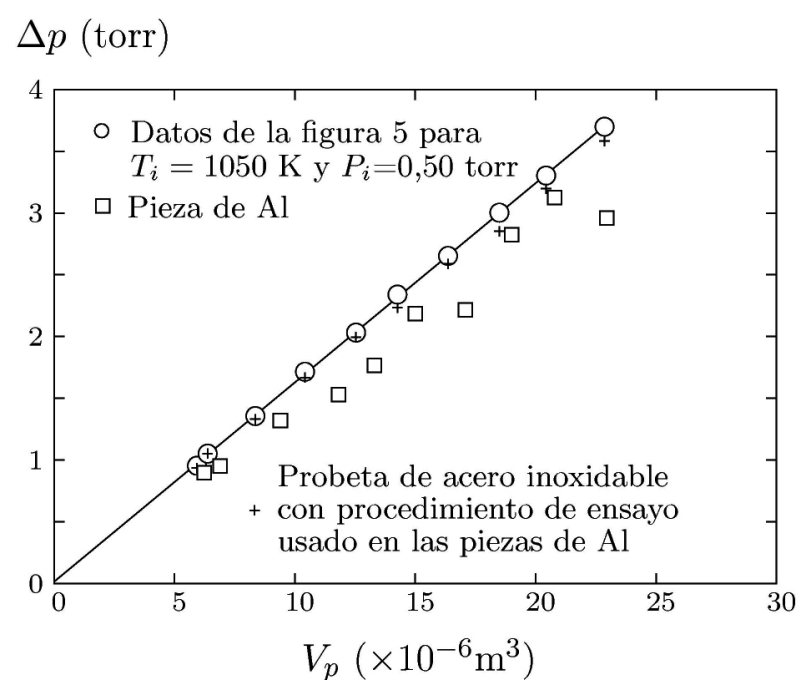

Figura 9. Comparación entre la curva de ajuste de la figura 5 , correspondiente a $T_{i}=1.050 \mathrm{~K} \mathrm{y}$ los incrementos de presión correspondientes a la liberación del gas de las piezas de aluminio. Los resultados correspondientes a la liberación del aire de las probetas de acero inoxidable siguiendo el procedimiento de ensayo establecido para piezas de aluminio, se incluyen en la figura con símbolo '+'.

Figure 9. Comparison between the fitting curve of figure 5 corresponding to $T_{i}=1050 \mathrm{~K}$ and pressure increases corresponding to the gas released from the aluminium parts. The results corresponding to the air released from the stainless steel test tubes when the test procedure applied to the aluminium castings is used, are included in the figure with ' + ' symbol.

'o') son relativamente pequeñas, lo que confirma la validez de la curva de ajuste correspondiente a la temperatura $T_{i}=1.050 \mathrm{~K}$ estabilizada, mostrada en la figura 5. La correlación de ajuste obtenida de dicha curva, con un coeficiente de regresión lineal, $R^{2}=0,9998$, es la siguiente:

$$
V=(6,169 \Delta p-0,094) \times 10^{-6}
$$

donde, $\Delta p$ es el incremento de presión en la cámara medido en torr y $V$ el volumen de aire atrapado medido en $\mathrm{m}^{3}$, referido a temperatura ambiente y presión atmosférica.

Por otro lado, la comparación mostrada en la figura 9 entre los resultados correspondientes a las piezas de aluminio y a las probetas de acero inoxidable muestra que, en general, el incremento de presión producido tras la fusión de la pieza de aluminio es ligeramente inferior al detectado cuando se libera la misma cantidad de gas desde la probeta de acero inoxidable. Esto, puede ser debido, en parte, a que el oxígeno existente en el aire de la pieza puede reaccionar, en mayor o menor medida, con el aluminio fundido formando óxidos y reduciendo de este modo la cantidad de gas liberado ${ }^{[21 ~ y ~ 22] . ~ E n ~ e l ~ f u t u r o, ~ s e ~ r e a l i z a r a ́ ~ u n ~ e s t u d i o ~ m a ́ s ~}$ detallado de los diferentes procesos de mezcla que pueden tener lugar durante la fusión del metal en el interior de la cámara de vacío.

Debe mencionarse que si la liberación del gas ocluido se produjese cuando la temperatura en la cámara aún no se hubiera estabilizado lo suficiente, lo que podría suceder, por ejemplo, si la liberación del gas se produce durante la fase rápida de calentamiento, habría que construir nuevas curvas de ajuste como las de la figura 5 para las mismas condiciones en las que se produzca la liberación del gas. Esto, será objeto de un trabajo posterior.

\subsection{Piezas de aluminio fabricadas mediante fundición a presión}

Como ejemplo de aplicación a piezas fundidas, se ha utilizado el equipo y el procedimiento de ensayo desarrollados para determinar el gas atrapado en dos piezas fabricadas con una máquina industrial de fundición por inyección a presión. La máquina empleada es de cámara fría horizontal con una fuerza de cierre de $1.500 \mathrm{kN}$. Las dimensiones de la pieza, de $4 \mathrm{~mm}$ de espesor, y una fotografía del molde empleado se pueden ver en la figura 10. El proceso de inyección se efectúa del modo que se indica a continuación (una descripción detallada del sistema de inyección de esta máquina se puede encontrar en la literatura ${ }^{[23]}$ ).

Un pistón empuja desde la cámara de inyección horizontal al metal fundido para introducirlo en la cavidad del molde. La cámara de inyección, de diámetro igual a $0,05 \mathrm{~m}$ y longitud igual a $0,265 \mathrm{~m}$, se llena, parcialmente, con una fracción de llenado igual a 0,51. En la fabricación de la primera pieza, el pistón inicia el movimiento empleando la ley de aceleración propuesta por López et al . ${ }^{[24]}$ hasta alcanzar una velocidad máxima igual a $1,07 \mathrm{~m} \mathrm{~s}^{-1}$ (velocidad máxima óptima obtenida por López et al. ${ }^{[25]}$, para la mencionada fracción de llenado), que se mantendrá constante durante todo el llenado de la cavidad del molde. En los trabajos de Zamora et al. ${ }^{[11 \text { y 23] }}$ se muestra, experimentalmente, que con esta ley de movimiento del pistón se consiguen reducir, significativamente, los fenómenos de atrapamiento de gas durante el proceso de inyección. En la radiografía mostrada en la figura 11 a) no se observan niveles de porosidad importantes (como referencia, en la figura $11 \mathrm{~b}$ ) se 
(dimensiones en $\mathrm{m}$ )

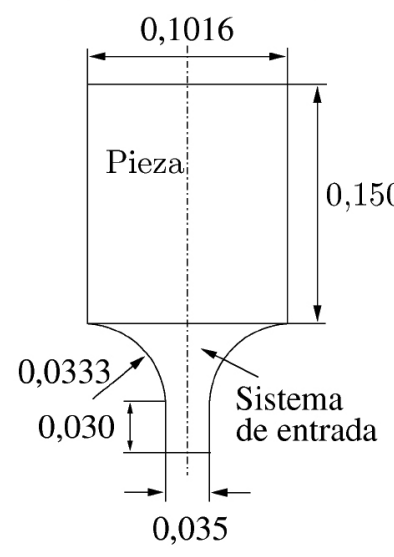

(a)

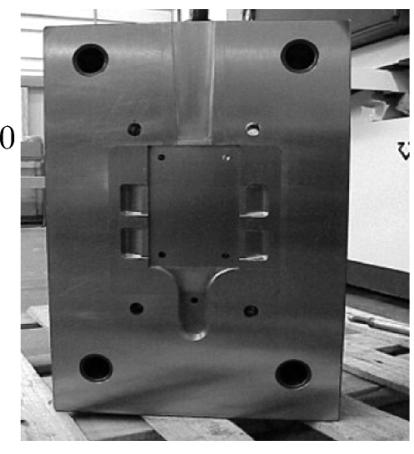

(b)
Figura 10. a) Geometría de las piezas cuya cantidad de gas se mide. b) Molde empleado en la máquina de fundición por inyección a presión para la fabricación de dichas piezas.

Figure 10. a) Geometry of the parts, whose gas is measured. b) Mould used in the diecasting machine to manufacture the castings.

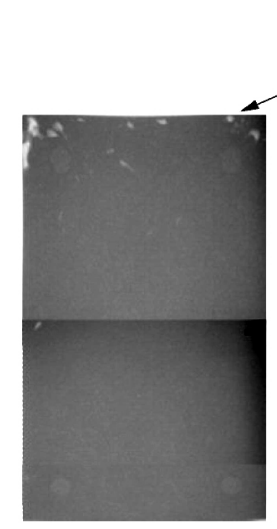

Pieza 1

(a)

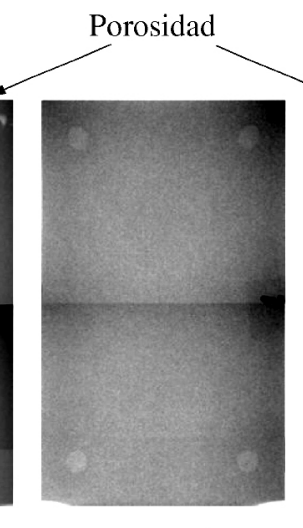

Pieza de referencia libre de poros

(b)

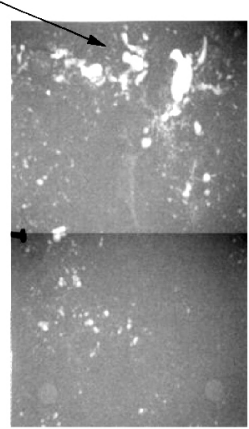

Pieza 2

(c)
Figura 11. Radiografías obtenidas para las piezas fabricadas mediante fundición por inyección a presión.

Figure 11. X-ray pictures obtained for the castings.

muestra la radiografía de una pieza totalmente libre de poros). En la fabricación de la segunda pieza, se emplea la misma ley de aceleración del pistón hasta alcanzar una velocidad máxima igual a $0,33 \mathrm{~m} \mathrm{~s}^{-1}$,
$P_{i}($ torr $)$

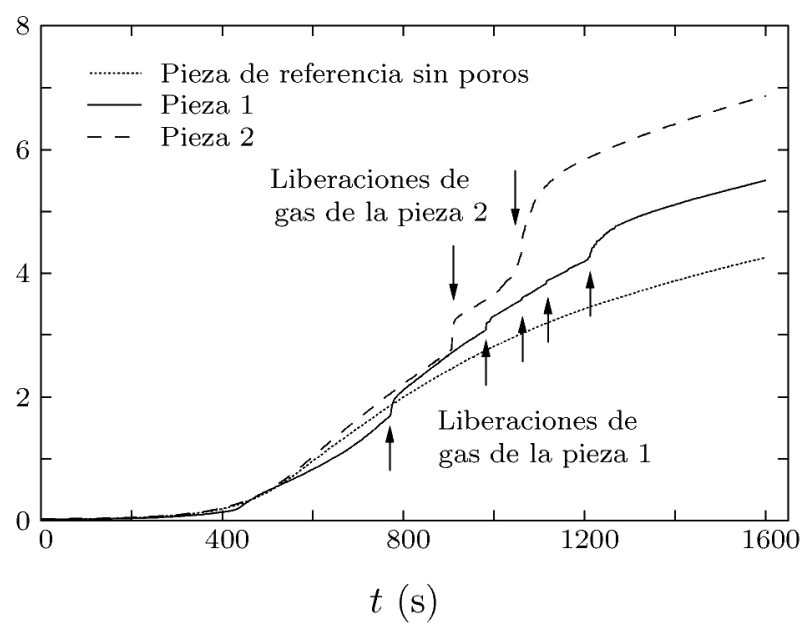

Figura 12. Registro de presión en la cámara de vacío obtenida durante la etapa final del ensayo de las piezas de la figura 11.

Figure 12. Pressure record in the vacuum chamber during the last step of testing the diecasting parts of figure. 11.

significativamente inferior al valor óptimo empleado en la primera pieza. La radiografía realizada a la segunda pieza (Fig. 11 c)) muestra claramente un importante incremento de los niveles de porosidad debido al aumento del atrapamiento de gas.

Cada pieza se ha fundido en el equipo de fusión al vacío construido, siguiendo el mismo procedimiento de ensayo descrito en la Sección 3.2. En la figura 12 se muestran los registros de presión, durante la liberación del gas atrapado, correspondientes a las dos piezas fabricadas. Junto a ellas se representa también el registro de presión obtenido durante el ensayo de la pieza de referencia libre de poros, en el que no se aprecia ningún salto de presión. Obsérvese que en la segunda pieza el salto de presión detectado es considerablemente mayor que el correspondiente a la primera pieza. Obsérvese, también, que a diferencia de las piezas de aluminio construidas con una cavidad interior, la liberación del gas en estas piezas obtenidas por fundición se puede producir en distintos intervalos de tiempo ya que el gas ocluido puede haber sido atrapado en distintas zonas de la pieza y a diferentes condiciones de presión. En la figura 12 se han indicado con flechas los instantes en los que se aprecia, a través del correspondiente salto de presión, alguna liberación de gas. La mayoría de las liberaciones que se observan se producen en ambos casos, en instantes posteriores a los $800 \mathrm{~s}$ de ensayo, es decir, cuando la temperatura, $T_{i}$, en la zona del 
crisol ha alcanzado una temperatura aproximadamente igual a la detectada durante la fusión de las piezas de la sección anterior (en torno a $1.050 \mathrm{~K}$ ).

El volumen de gas liberado obtenido de la ecuación (3), correspondiente a los incrementos de presión detectados, es de $3,753 \times 10^{-6} \mathrm{~m}^{3}$ (lo que corresponde al 6,15\% del volumen total de la pieza), para la primera pieza, y de $9,962 \times 10^{-6} \mathrm{~m}^{3}(16,34 \%)$ para la segunda.

\section{CONCLUSIONES}

Se ha diseñado y fabricado un equipo de fusión al vacío que permite la cuantificación del gas atrapado en piezas fabricadas por fundición. Los principales inconvenientes de este tipo de equipos se han abordado con cierto éxito, mejorando la operatividad de equipos previamente fabricados y permitiendo alcanzar temperaturas suficientemente altas para evaluar piezas de aleaciones de aluminio con relativa precisión. Se ha establecido y aplicado una metodología para la verificación de este tipo de equipos, basada en la liberación de cantidades de aire conocidas procedentes de probetas calibradas, obteniendo curvas de ajuste para diferentes condiciones de presión y temperatura de la cámara de vacío. Los resultados obtenidos muestran un buen grado de concordancia con las predicciones de tipo teórico. Se ha establecido y validado un procedimiento de ensayo que permite determinar con precisión la cantidad de gas ocluido en piezas de aluminio a partir de las curvas de ajuste obtenidas. La precisión del equipo en condiciones reales de operación ha sido evaluada mediante la fusión de 10 piezas de aluminio construidas con valores conocidos de gas ocluido. Finalmente, el equipo se ha aplicado a la medición del gas atrapado en dos piezas obtenidas mediante un proceso de fundición a presión. Los resultados obtenidos confirman la utilidad del equipo construido, lo que sin duda ayudará a seguir mejorando y optimizando las condiciones de operación en los procesos de fundición para minimizar la cantidad de gas atrapado y así los niveles de porosidad en las piezas fundidas.

\section{Agradecimientos}

Los autores desean expresar su agradecimiento al Ministerio de Educación y Ciencia español por la financiación concedida a través de los proyectos DPI2004-08198 y DPI2006-07047, y a D. Miguel López Almorox de la empresa Microtest S.A. por su colaboración en la fabricación del equipo.

\section{REFERENCIAS}

[1] ISO 10049, Aluminium Alloy Casting-Visual Method for Assessing the Porosity, 1992.

[2] ASTM E155-00, Standard Reference Radiographs for Inspection of Aluminum and Magnesium Castings, ASTM International, 2000.

[3] ASTM E1441-00, Standard Guide for Computed Tomography (CT) Imaging, ASTM, 2005.

[4] S. Palanisamy, R. Nagarajah, y P. Iovenitti, Transactions of the Die Casting in the Heartland, Indianapolis, EE. UU., 2003, Ed. NADCA, paper T03-22.

[5] T.E. Prucha y R. Nath, Proc. SAE World Congress, Detroit EE. UU., 2003, Ed. SAE International, paper 2003-01-0436.

[6] L. Garber y A.B. Draper, Trans. 10th SDCE Int. Die Casting Exposition and Congress, St. Louis, EE. UU., 1979, Ed. NADCA, Paper G-T79022.

[7] L. Luis-Martín, T. Rosa y J. Pérez, Rev. Metal. Madrid 15 (1979) 221-234.

[8] J.R. Brevick y P.S. Cheng, Trans. 18th Int. Die Casting Cong. Exp., Indianapolis (USA), 1995, Ed. NADCA, paper T95-045.

[9] K. S. Anastasiou, P. I. Mech. Eng. B-J Eng., 216 (2002) 969-977.

[10] V.D. Tsoukalas, Int. J. Cast. Metal. Res. 15 (2003) 581-588.

[11] R. Zamora, J.J. Hernández-Ortega, F. Faura, J. López y J. Hernández, J. Manuf. Sci. E-T ASME. 130 (2008).

[12] Y.J. Huang, B.H. Hu, I. Pinwill, W. Zhou y D.M.R. Taplin, Mater. Manuf Process. 15 (2000) 97-105.

[13] S.T. Mcclain, MS Degree Thesis, Mississippi State University, 1997.

[14] L. Luis y J.I. Robla, Trans. 12th Int. Die Casting Cong. and Exp., Minneapolis, EE. UU., 1983, Ed. NADCA, paper G-T83-052.

[15] J.I. Robla y L. Luis, J. Vac. Sci. Technol. 4 (1987) 2.693-2.695.

[16] L. Luis y J.I. Robla, Trans. 14th Int. Die Casting Congress and Exposition, Toronto (Canada), 1987, Ed. NADCA, paper G-T87-029.

[17] L. Wang, W. Alguine, M. Gershenzon, G. Savage y K. Rogers, Trans. Die Casting in the Heartland, Indianapolis, EE. UU., 2003, Ed. NADCA, paper T03-048.

[18] R.D. Webb, 1999. Patent Number 55,987,053.

[19] R. Zamora, Tesis doctoral, Escuela Técnica Superior de Ingeniería Industrial, Universidad Politécnica de Cartagena, 2005. 
[20] X. Hao, J. Gu, N. Chen, W. Zhang, y X. Zuo, Appl. Therm. Eng. (2007) doi: 10.1016/j.applthermaleng.2007.12.007.

[21] Specialty Handbook,1996, Aluminum and Aluminum alloys, ASM International, pp. 199-206.

[22] G. Bar-Meir, J. Eng. Mater-T ASME 117 (1995) 215-219.
[23] R. Zamora, F. Faura, J. López y J. Hérnandez, Int. J. Ad. Manuf. Tech. 33(2007) 266-277.

[24] J. López, J. Hernández, F. Faura y G. Trapaga, J. Fluid. Eng-T ASME, 122(2) (2000) 349-356.

[25] J. López, F. Faura, J. Hernández y P. Gómez, J. Manuf. Sci. E-T ASME 125(3) (2003) 529. 537. 